\title{
Gravitational Wave Detectors on the Ground and in Space
}

\author{
Karsten Danzmann* \\ *Institut für Atom- und Molekülphysik, Universität Hannover, \\ and Max-Planck Institut für Quantenoptik, Außenstelle Hannover, \\ Callinstr. 38, D-30167 Hannover, Germany
}

\begin{abstract}
Small prototypes of gravitational wave detectors have been under development for over 30 years. But it is only now that we have the necessary technology available to build large instruments with good sensitivity. After several years of construction, the first ground-based interferometers will go into operation in 2001 and a space-based detector is expected to be launched in 2010. These instruments will complement each other because the gravitational wave spectrum extends over many decades in frequency. Ground-based detectors can only observe the audio-frequency regime above $1 \mathrm{~Hz}$, while sources in the low-frequency regime are only accessible from space because of the unshieldable background of local gravitational noise on the ground.
\end{abstract}

\section{INTRODUCTION}

If a body changes its shape, the resulting change in the force field will make its way outward at the speed of light. As early as 1805, Laplace, in his famous Traité de Mécanique Céleste stated that, if Gravitation propagates with finite speed, the force in a binary star system should not point along the line connecting the stars, and the angular momentum of the system must slowly decrease with time. In modern language we would say the binary star is losing energy and angular momentum by emitting gravitational waves. In 1993, Hulse and Taylor were awarded the Nobel prize in physics for the indirect proof of the existence of Gravitational Waves using exactly this kind of observation on the binary pulsar PSR 1913+16. A direct detection of gravitational waves has not been achieved up to this day.

Einstein's paper on gravitational waves was published in 1916, but it was not before the late 1950s that it was rigorously proven that gravitational radiation was in fact a physically observable phenomenon, that gravitational waves carry energy and that, as a result, a system that emits gravitational waves should lose energy.

General Relativity replaces the Newtonian picture of Gravitation by a geometric one that is very intuitive if we are willing to accept the fact that space and time do not have an independent existence but rather are in intense interaction with the physical world. Massive bodies produce "indentations" in the fabric of spacetime,

CP551, Atomic Physics 17, edited by E. Arimondo, P. DeNatale, and M. Inguscio (C) 2001 American Institute of Physics 1-56396-982-3/01/\$18.00 
and other bodies move in this curved spacetime taking the shortest path, much like a system of billard balls on a springy surface. In fact, the Einstein field equations relate mass (energy) and curvature in just the same way that Hooke's law relates force and spring deformation, or phrased somewhat poignantly: spacetime is an elastic medium.

If a mass distribution moves in an asymmetric way, then the spacetime indentations travel outwards as ripples in spacetime called gravitational waves. Gravitational waves are fundamentally different from the familiar electromagnetic waves. While electromagnetic waves, created by the acceleration of electric charges, propagate IN the framework of space and time, gravitational waves, created by the acceleration of masses, are waves of the spacetime fabric ITSELF.

Unlike charge, which exists in two polarities, mass always come with the same sign. This is why the lowest order asymmetry producing electro-magnetic radiation is the dipole moment of the charge distribution, whereas for gravitational waves it is a change in the quadrupole moment of the mass distribution. Hence those gravitational effects which are spherically symmetric will not give rise to gravitational radiation. A perfectly symmetric collapse of a supernova will produce no waves, a non-spherical one will emit gravitational radiation. A binary system will always radiate.

Gravitational waves distort spacetime, in other words they change the distances between free macroscopic bodies. A gravitational wave passing through the Solar System creates a time-varying strain in space that periodically changes the distances between all bodies in the Solar System in a direction that is perpendicular to the direction of wave propagation. The main problem is that the relative length change due to the passage of a gravitational wave is exceedingly small. This is not to mean that gravitational waves are weak in the sense that they carry little energy. On the contrary, a supernova in a not too distant galaxy will drench every square meter here on earth with kilowatts of gravitational radiation intensity. The resulting length changes, though, are very small because spacetime is an extremely stiff elastic medium so that it takes extremely large energies to produce even minute distortions.

\section{SOURCES OF GRAVITATIONAL WAVES}

The gravitational wave sources observable from the ground and from space space are profoundly different, due to the very different frequency ranges in which they emit.

\section{A Sources for ground-based detectors}

Strong bursts from supernovae have always been a prime target for ground-based detectors. It is becoming increasingly clear that there is a large variety of supernovae, and many low-luminosity ones (like SN 1987 a) are missed in surrounding 
galaxies. Computer simulations are still not able to predict realistically what will happen in a gravitational collapse with high angular momentum, which is the situation likely to lead to gravitational radiation. Pulsar evidence [1] now sugggests that the mean space velocity of pulsars is three times higher than had previously been estimated. This linear velocity must come from some non-axisymmetric asymmetry in the gravitational collapse, and this would also enhance one's expectations of gravitationla radiation

Coalescing binary systems have long been among the best understood sources of gravitational radiation. Observations of pulsars like the Hulse-Taylor pulsar PSR $1913+16$ are suggesting that in order to see an event rate of coalescences of such systems of more than one per year, one has to observe out to a distance of more than $100 \mathrm{Mpc}$. Recently though, theoretical studies of binary evolution [2] have suggested that there should be a large population of very tight neutron star binaries that have such short inspiral times that the chances of seeing one at any particular time in our galaxy are very small, but the coalescence rate integrated over time in our galaxy could be factors of ten or more larger than before.

Pulsars and accreting neutron stars are promising sources that would produce nearly monochromatic gravitational wave spectra. The spindown rate of observed pulsars sets an upper limit to their emission of gravitational radiation. The assumption that gravitational radiation is the dominant source of energy loss is not unreasonable at least for young pulsars with significant irregularities in their shape. In this case there might well be several in our neighborhood.

\section{B Sources for space-based detectors}

The two main categories of gravitational waves sources for space-based detectors like LISA are the galactic binaries and the massive black holes (MBHs) expected to exist in the centres of most galaxies.

Because the masses involved in typical binary star systems are small (a few solar masses), the observation of binaries is limited to our Galaxy. Galactic sources that can be detected by LISA include a wide variety of binaries, such as pairs of close white dwarfs, pairs of neutron stars, neutron star and black hole (5$-20 M_{\odot}$ ) binaries, pairs of contacting normal stars, normal star and white dwarf (cataclysmic) binaries, and possibly also pairs of black holes. It is likely that there are so many white dwarf binaries in our Galaxy that they cannot be resolved at frequencies below $10^{-3} \mathrm{~Hz}$, leading to a confusion-limited background. Some galactic binaries are so well studied, especially the X-ray binary $4 \mathrm{U} 1820-30$, that it is one of the most reliable sources. If LISA would not detect the gravitational waves from known binaries with the intensity and polarisation predicted by General Relativity, it will shake the very foundations of gravitational physics.

The main objective of the LISA mission, however, is to learn about the formation, growth, space density and surroundings of massive black holes (MBHs). There is now compelling indirect evidence for the existence of MBHs with masses of $10^{6}$ 
to $10^{8} M_{\odot}$ in the centres of most galaxies, including our own. The most powerful sources are the mergers of MBHs in distant galaxies, with amplitude signal-tonoise ratios of several thousand for $10^{6} M_{\odot}$ black holes. Observations of signals from these sources would test General Relativity and particularly black-hole theory to unprecedented accuracy. Not much is currently known about black holes with masses ranging from about $100 M_{\odot}$ to $10^{6} M_{\odot}$. LISA can provide unique new information throughout this mass range.

\section{COMPLEMENTARITY OF DETECTION ON THE GROUND AND IN SPACE}

Astronomical observations of electromagnetic waves cover a range of 20 orders of magnitude in frequency, from ULF radio waves to high-energy gamma-rays. Almost all of these frequencies (except for visible and radio) cannot be detected from the Earth, and therefore it is necessary to place detectors optimised for a particular frequency range (e.g. radio, infrared, ultraviolet, X-ray, gamma-ray) in space.

The situation is similar for gravitational waves. The range of frequencies spanned by ground- and space-based detectors, as shown schematically in Figure 1, is comparable to the range from high frequency radio waves up to X-rays. Ground-based detectors will never be sensitive below about $1 \mathrm{~Hz}$, because of terrestrial gravitygradient noise. A space-based detector is free from such noise and can be made very large, thereby opening the range from $10^{-4} \mathrm{~Hz}$ to $1 \mathrm{~Hz}$, where both the most certain and the most exciting gravitational-wave sources radiate most of their power.

The importance of low frequencies is a consequence of Newton's laws. For systems involving solar-mass objects, lower frequencies imply larger orbital radii, and the range down to $10^{-4} \mathrm{~Hz}$ includes sources with the typical dimensions of many galactic neutron star binaries, cataclysmic binaries, and some known binaries. These are the most certain sources. For highly relativistic systems, where the orbital velocities approach the speed of light, lower frequencies imply larger masses $(M \propto 1 / f)$, and the range down to $10^{-4} \mathrm{~Hz}$ reaches masses of $10^{7} M_{\odot}$, typical of the black holes that are believed to exist in the centres of many, if not most, galaxies. Their formation and coalescences could be seen anywhere in the Universe and are among the most exciting of possible sources. Detecting them would test the strong-field limit of gravitational theory and illuminate galaxy formation and quasar models.

For ground-based detectors, on the other hand, their higher frequency range implies that even stellar-mass systems can last only for short durations, so these detectors will mainly search for short-lived catastrophic events (supernovae, coalescing neutron-star binaries). Normally, several detectors are required for directional information. If such events are not detected in the expected way, this will upset the astrophysical models assumed for such systems, but not necessarily contradict gravitation theory.

By contrast, if a space-based interferometer does not detect the gravitational waves from known binaries with the intensity and polarisation predicted by Gen- 


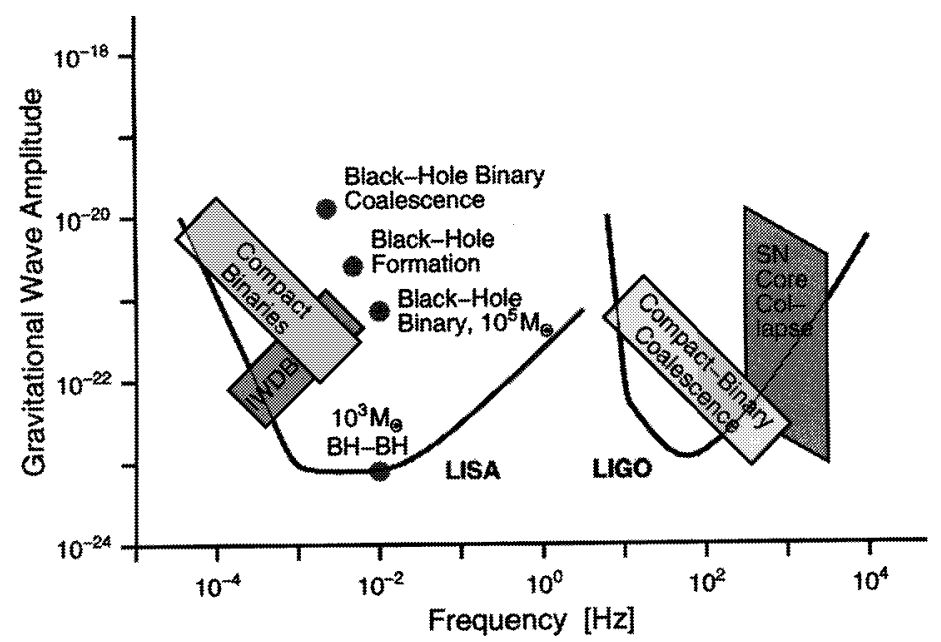

FIGURE 1. Comparison of frequency range of sources for ground-based and space-based gravitational wave detectors. Only a few typical sources are indicated, ranging in frequency from the $\mathrm{kHz}$ region of supernovae and final mergers of binary stars down to $\mathrm{mHz}$ events due to formation and coalescence of supermassive black holes, compact binaries and interacting white dwarf binaries. The sources shown are in two clearly separated regimes: events in the range from, say, $10 \mathrm{~Hz}$ to several $\mathrm{kHz}$ (and only these will be detectable with terrestrial antennas), and a low-frequency regime, $10^{-4}$ to $10^{-1} \mathrm{~Hz}$, accessible only with a space project. Sensitivities of LISA for periodic sources, and of (the "Advanced") LIGO for burst sources, are indicated.

eral Relativity, it will undermine the very foundations of gravitational physics. Furthermore, even some highly relativistic events, such as massive black hole coalescences with masses below $10^{5} M_{\odot}$, last roughly a year or longer. This allows a single space-based detector to provide directional information as it orbits the Sun during the observation.

Both ground- and space-based detectors will also search for a cosmological background of gravitational waves. Since both kinds of detectors have similar energy sensitivities, their different observing frequencies are ideally complementary: observations can supply crucial spectral information.

The space-based interferometer proposal has the full support of the groundbased detector community. Just as it is important to make observations at radio, optical, X-ray, and all other electromagnetic wavelengths, so too is it important to cover different gravitational-wave frequency ranges. Ground-based and space-based observations will therefore complement each other in an essential way. 


\section{GROUND-BASED DETECTORS}

The highest frequencies expected for the emission of strong gravitational waves are around $10 \mathrm{kHz}$ because a gravitational wave source cannot emit strongly at periods shorter than the light travel time across its gravitational radius. At frequencies below $1 \mathrm{~Hz}$, observations on the ground are impossible because of an unshieldable background due to Newtonian gravity gradients on the earth. These two frequencies define the limits of the high-frequency band of gravitational radiation, mainly populated by signals from neutron star and stellar mass black hole binaries. This band is the domain of ground-based detectors: laser interferometers and resonant-mass detectors.

\section{A Resonant-mass detectors}

The history of attempts to detect gravitational waves began in the 1960s with the famous bar experiments of Joseph Weber [7]. A resonant-mass antenna is, in principle, a simple object. It consists of a solid body that during the passage of a gravitational wave gets excited similarly to being struck with a hammer, and then rings like a bell.

The solid body traditionally used to be a cylinder, that is why resonant-mass detectors are usually called bar detectors. But in the future we may see very promising designs in the shape of a sphere or sphere-like object like a truncated icosahedron. The resonant mass is usually made from an aluminum alloy and has a mass of several tons. Occasionally, other materials are used, e.g. silicon, sapphire or niobium.

The first bar detectors were operated at room temperature, but the present generation of bars is operating below liquid-helium temperature. The next generation, which is already under construction (NAUTILUS in Frascati, AURIGA in Legnaro), will operate at a temperature around $50 \mathrm{mK}$.

Resonant-mass detectors are equipped with transducers that monitor the complex amplitudes of one or several of the bar's vibrational modes. A passing gravitational wave changes these amplitudes due to its frequency content near the normal mode frequencies. Present-day resonant mass antennas are fairly narrowband devices, with bandwidths of only a few $\mathrm{Hz}$ around centre-frequencies in the $\mathrm{kHz}$ range. With improved transducer designs in the future, we may see the bandwidth improve to $100 \mathrm{~Hz}$ or better.

The sensitivities of bar antennas have steadily improved since the first experiments of Joe Weber. Currently, we see a network of antennas at CERN, Frascati, Louisiana State, Legnaro and Perth operating at an rms noise level equivalent to $h_{\mathrm{rms}} \approx 6 \times 10^{-19}$. In the first decade of the next millennium, planned sphere-like detectors operating near the standard quantum limit may reach burst sensitivities below $10^{-21}$ in the $\mathrm{kHz}$ range [8]. 


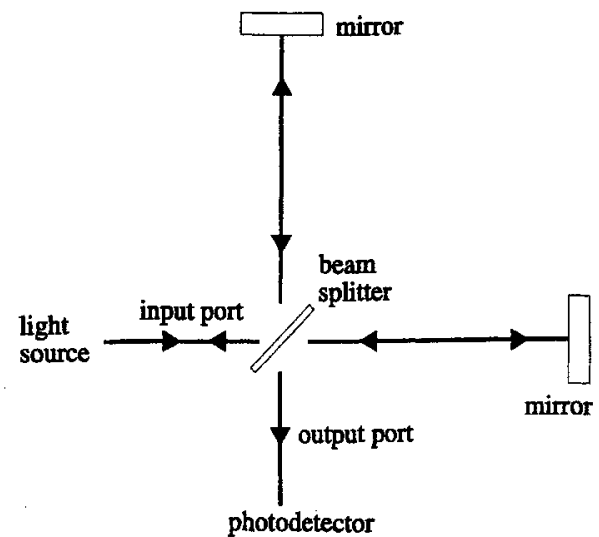

FIGURE 2. Schematic of a two-arm Michelson interferometer. Interference of the two returning beams on the photodetector gives a measure of their relative phase. Any variation in the mirror distances caused by a passing gravitational wave will modulate this phase signal. By having both arms fed from the same light source, the phase noise inherent to a non-ideal source is the same in each arm and cancels.

\section{B Laser Interferometers}

Although the seeds of the idea can be found in early papers by Pirani [9] and Gertsenshtein and Pustovoit [10], it was really in the early 1970s that the idea emerged that laser interferometers might have a better chance of detecting gravitational waves, mainly promoted by Weiss [11] and Forward [12].

A Michelson interferometer measures the phase difference between two light fields having propagated up and down two perpendicular directions, i.e. essentially the length difference between the two arms. This is the quantity that would be changed by a properly oriented gravitational wave. The phase difference measured can be increased by increasing the armlength, or, equivalently, the interaction time of the light with the gravitational wave, up to an optimum for an interaction time equal to half a gravitational wave period. For a gravitational wave frequency of $100 \mathrm{~Hz}$ this corresponds to five milliseconds or an armlength of $750 \mathrm{~km}$. On the ground it is clearly impractical to build such large interferometers, but there are ways to increase the interaction time without increasing the physical armlength beyond reasonable limits. Several variants have been developed, all of them relying on storing and enhancing the laser light, or the gravitational-wave induced sidebands, or both. The technology and techniques for such interferometers have now been under development for nearly 30 years. Figure 3 gives an impression of the wide international scope of the interferometer efforts. After pioneering work at MIT, other groups at Munich/Garching, at Glasgow, then Caltech, Paris/Orsay, Pisa, and later in Japan, also entered the scene. Their prototypes range from a few meters up to 30,40 , and even $100 \mathrm{~m}$.

Today, these prototype interferometers are routinely operating at a displacement noise level of the order $10^{-19} \mathrm{~m} / \sqrt{\mathrm{Hz}}$ over a frequency range from $200 \mathrm{~Hz}$ to $1000 \mathrm{~Hz}$, corresponding to an rms gravitational-wave amplitude noise level of $h_{\mathrm{rms}} \approx 10^{-19}$.

Plans for kilometer-size interferometers have been developed for the last 15 years. All of these large-scale projects will use low-noise Nd:YAG lasers (wavelength $1.064 \mu \mathrm{m})$, pumped with laser diodes, just as is intended for the space detector 


\begin{tabular}{|l|c|c|c|c|c|c|c|c|}
\hline $\begin{array}{l}\text { Country: } \\
\text { Institute: }\end{array}$ & $\begin{array}{c}\text { USA } \\
\text { MIT }\end{array}$ & $\begin{array}{c}\text { USA } \\
\text { Caltech }\end{array}$ & $\begin{array}{c}\text { GER } \\
\text { MPQ }\end{array}$ & $\begin{array}{c}\text { GBR } \\
\text { Glasgow }\end{array}$ & $\begin{array}{c}\text { FRA } \\
\text { CNRS }\end{array}$ & $\begin{array}{c}\text { ITA } \\
\text { INFN }\end{array}$ & $\begin{array}{l}\text { JPN } \\
\text { ISAS }\end{array}$ & $\begin{array}{c}\text { JPN } \\
\text { NAO }\end{array}$ \\
\hline
\end{tabular}

Prototypes:

\begin{tabular}{|l|c|c|c|c|c|c|c|c|}
\hline Start: & 1972 & 1980 & 1975 & 1977 & 1983 & 1986 & 1986 & 1991 \\
\hline Laser: & $\mathrm{Ar}^{+}$ & $\mathrm{Ar}^{+}$ & $\mathrm{Ar}^{+}$ & $\mathrm{Ar}^{+}$ & $\left(\mathrm{Ar}^{+}\right)$ & $\left(\mathrm{Ar}^{+}\right)$ & $\mathrm{Ar}^{+}$ & $\mathrm{YAG}$ \\
\hline Arm length $\ell:$ & $40 \mathrm{~m}$ & $30 \mathrm{~m}$ & $10 \mathrm{~m}$ & $0.5 \mathrm{~m}$ & & $100 \mathrm{~m}$ & $20 \mathrm{~m}$ \\
\hline $\begin{array}{c}\text { Strain sensitivity } \\
\tilde{h}\left[\mathrm{~Hz}^{-\frac{1}{2}}\right]:\end{array}$ & $\begin{array}{c}1 \cdot 10^{-20} \\
1995\end{array}$ & $\begin{array}{c}11 \cdot 10^{-20} \\
1986\end{array}$ & $\begin{array}{c}6 \cdot 10^{-20} \\
1992\end{array}$ & & & $8 \cdot 10^{-20}$ & $2 \cdot 10^{-18}$ \\
\hline
\end{tabular}

Large Interferometric Detectors:

\begin{tabular}{|c|c|c|c|c|c|c|c|c|}
\hline Planning (start): & 1982 & 1984 & 1985 & 1986 & 1986 & 1986 & 1987 & 1994 \\
\hline Arm length $\ell:$ & $\begin{array}{l}4 \mathrm{~km} \\
2 \mathrm{~km}\end{array}$ & $4 \mathrm{~km}$ & \multicolumn{2}{|c|}{$600 \mathrm{~m}$} & \multicolumn{2}{|c|}{$3 \mathrm{~km}$} & \multicolumn{2}{|c|}{$300 m$} \\
\hline $\begin{array}{l}\text { Site } \\
\text { (State) }\end{array}$ & \multicolumn{2}{|c|}{$\begin{array}{l}\text { Hanford Livingston } \\
\begin{array}{ll}\text { (WA) } & \text { (LA) }\end{array}\end{array}$} & \multicolumn{2}{|c|}{$\begin{array}{l}\text { Hannover } \\
\text { GER }\end{array}$} & \multicolumn{2}{|c|}{$\begin{array}{l}\text { Pisa } \\
\text { ITA }\end{array}$} & \multicolumn{2}{|c|}{$\begin{array}{l}\text { Mitaka } \\
\text { JPN }\end{array}$} \\
\hline Cost $\left(10^{6}\right.$ US\$): & \multicolumn{2}{|c|}{292} & \multicolumn{2}{|c|}{7} & \multicolumn{2}{|c|}{90} & \multicolumn{2}{|c|}{15} \\
\hline Project name: & \multicolumn{2}{|c|}{ LIGO } & \multicolumn{2}{|c|}{ GEO 600} & \multicolumn{2}{|c|}{ VIRGO } & \multicolumn{2}{|c|}{ TAMA 300} \\
\hline
\end{tabular}

FIGURE 3. Funded ground-based interferometric gravitational wave detectors: List of prototypes (upper part) and long-baseline projects (lower part).

LISA, which will greatly benefit from their efforts for achieving extreme stability and high overall efficiency. In contrast to LISA, all ground-based detectors will employ a technique called power recycling to enhance the laser power circulating in the interferometer over the power available out of the laser. To achieve this, the interferometer output is kept dark by a control system, so that all the laser power is going back to the input, where it is sent back into the onterferometer by a power recycling mirror, forming a resonant Fabry-Perot cavity that consists of the power recycling mirror as incoupling mirror and the whole locked interferometer as end mirror.

The US project LIGO calls for two facilities at two widely separated sites [13], one in Hanford, Washington, and one in Livingston, Louisiana. Both will house a $4 \mathrm{~km}$ interferometer, Hanford an additional $2 \mathrm{~km}$ interferometer. At both sites groundwork and construction have been finished, and vacuum tests (of the "world's largest vacuum chamber") were successful. The installation of optics and suspensions has been completed and laser light is on site. In Hanford, both $2 \mathrm{~km}$ arm cavities were locked to the laser for extended periods, and currently the power-recycled vertex interferometer without the long arms is resonating. Louisiana is scheduled to follow with a certain time delay and commissioning of both interferometers is expected to begin in 2001 with routine data taking in 2002.

In the French-Italian project VIRGO, being built near Pisa, an elaborate seismic isolation system will allow this project to measure down to a frequency of $10 \mathrm{~Hz}$ or even below [14]. Construction of the center building and the mode cleaner is 
finished and the ground-work for the long arms has begun. A laser is on site, the central vacuum system is in place, and currently the mode-cleaner cavity is going into operation. VIRGO will follow about a year after LIGO, with routine data taking planned to commence in 2003.

A British-German collaboration has de-scoped the project of a $3 \mathrm{~km}$ antenna to a length of only $600 \mathrm{~m}$ : GEO 600 [15]. It will employ advanced optical techniques to make up for the shorter arms. Ground work and construction at the site near Hannover are completed, the vacuum system tested. Most of the optics have been installed and both mode-cleaners are locking. Currently, the east-west arm of GEO 600 is going into operation. The schedule is comparable to LIGO, with routine data taking planned for 2002.

In Japan, after a merger of efforts at ISAS and other institutions, construction and vacuum verification of a common $300 \mathrm{~m}$ project called TAMA 300 [16] is completed. TAMA is now operating as an interferometer, although not with final sensitivity yet, because power recycling has not yet been installed.

Not included in Figure 3 is the (not yet funded) Australian project of a $500 \mathrm{~m}$ detector to be built near Perth. The site would allow later extension to $3 \mathrm{~km}$ arms.

LIGO, VIRGO, GEO 600 and TAMA 300 are scheduled to be completed by the end of this century. Observations may begin in 2001 or 2002, although the sensitivity of the first stage detectors may be only marginally sufficient to detect gravitational waves. However, step-by-step improvements will be made, until the network finally reaches the advanced detector sensitivity sometime between 2005 and 2010 . At that point, one can be confident that signals will be observed from sources such as supernovae, compact binary coalescences and pulsars, unless something is fundamentally wrong with our current estimates of their strength and distribution.

\section{PULSAR TIMING}

Man-made gravitational wave detectors operate by detecting the effect of gravitational waves on the apparatus. It is also possible to detect gravitational waves by observing their effect on electromagnetic waves as they travel to us from astronomical objects. Such methods of detection are like "one-arm interferometers" the second arm is not needed if there is another way to provide a reference clock stable enough to sense the changes in propagation time produced by gravitational waves.

Pulsar timing makes use of the fact that the pulsar is a very steady clock. If we have a clock on the Earth that is as stable as the pulsar, then irregularities in the arrival times of pulses that are larger than expected from the two "clocks" can be attributed to external disturbances, and in particular possibly to gravitational waves. Since the physics near a pulsar is poorly known, it might be difficult to prove that observed irregularities are caused by gravitational waves. But where irregularities are absent, this provides an upper limit to the gravitational wave field. This is how such observations have been used so far. 
All pulsars slow down, and a few have shown systematic changes in the slowing down rate. Therefore, it is safer to use random irregularities in the pulsar rate as the detection criterion, rather than systematic changes. Such random irregularities set limits on random gravitational waves: the stochastic background.

The arrival times of individual pulses from most pulsars can be very irregular Pulsar periods are stable only when averaged over considerable times. The longer the averaging period, the smaller are the effects of this intrinsic irregularity. Therefore, pulsar timing is used to set limits on random gravitational waves whose period is of the same order as the total time the pulsar has been observed, from its discovery to the present epoch. Millisecond pulsars seem to be the most stable over these long periods, and a number of them are being used for these observations.

The best limits come from the first discovered millisecond pulsar, PSR $1937+21$ At a frequency of approximately 1 per 10 years the pulsar sets an upper limit on the energy density of the gravitational wave background of $\Omega_{\mathrm{GW}}<10^{-7}[17]$. This is in an ultra-low frequency range that is $10^{5}$ times lower than the LISA band and $10^{10}$ times lower than the ground-based band. If one believes a theoretical prediction of the spectrum of a cosmic gravitational wave background, then one can extrapolate this limit to the other bands. But this may be naive, and it is probably wiser to regard observations in the higher-frequency bands as independent searches for a background.

More-recently discovered millisecond pulsars are also being monitored and will soon allow these limits to be strengthened. If irregularities are seen in all of them at the same level, and if these are independent of the radio frequency used for the observations, then that will be strong evidence that gravitational waves are indeed responsible.

These observations have the potential of being extended to higher frequencies by directly cross-correlating the data of two pulsars. In this way one might detect a correlated component caused by gravitational waves passing the Earth at the moment of reception of the radio signals from the two pulsars. Higher frequencies are accessible because the higher intrinsic timing noise is reduced by the crosscorrelation. Again, seeing the effect in many pairs of pulsars independently of the radio frequency would be strong evidence for gravitational waves.

\section{SPACECRAFT TRACKING}

Precise, multi-frequency transponding of microwave signals from interplanetary probes, such as the ULYSSES, GALILEO and CASSINI spacecraft, can set upper limits on low-frequency gravitational waves. These appear as irregularities in the time-of-communication residuals after the orbit of the spacecraft has been fitted. The irregularities have a particular signature. Searches for gravitational waves have produced only upper limits so far, but this is not surprising: their sensitivity is far short of predicted wave amplitudes. This technique is inexpensive and well worth pursuing, but will be limited for the forseeable future by some combination 
of measurement noise, the stability of the frequency standards, and the uncorrected parts of the fluctuations in propagation delays due to the interplanetary plasma and the Earth's atmosphere. Consequently, it is unlikely that this method will realise an $r m s$ strain sensitivity much better than $10^{-17}$, which is six orders of magnitude worse than that of a space-based interferometer.

\section{THE LISA MISSION}

The LISA mission comprises three identical spacecraft located $5 \times 10^{6} \mathrm{~km}$ apart forming an equilateral triangle. LISA is basically a giant Michelson interferometer placed in space, with a third arm added to give independent information on the two gravitational wave polarizations, and for redundancy. The distance between the spacecraft - the interferometer arm length - determines the frequency range in which LISA can make observations; it was carefully chosen to allow for the observation of most of the interesting sources of gravitational radiation. The centre of the triangular formation is in the ecliptic plane, $1 \mathrm{AU}$ from the Sun and $20^{\circ}$ behind the Earth. The plane of the triangle is inclined at $60^{\circ}$ with respect to the ecliptic. These particular heliocentric orbits for the three spacecraft were chosen such that the triangular formation is maintained throughout the year with the triangle appearing to rotate about the centre of the formation once per year.

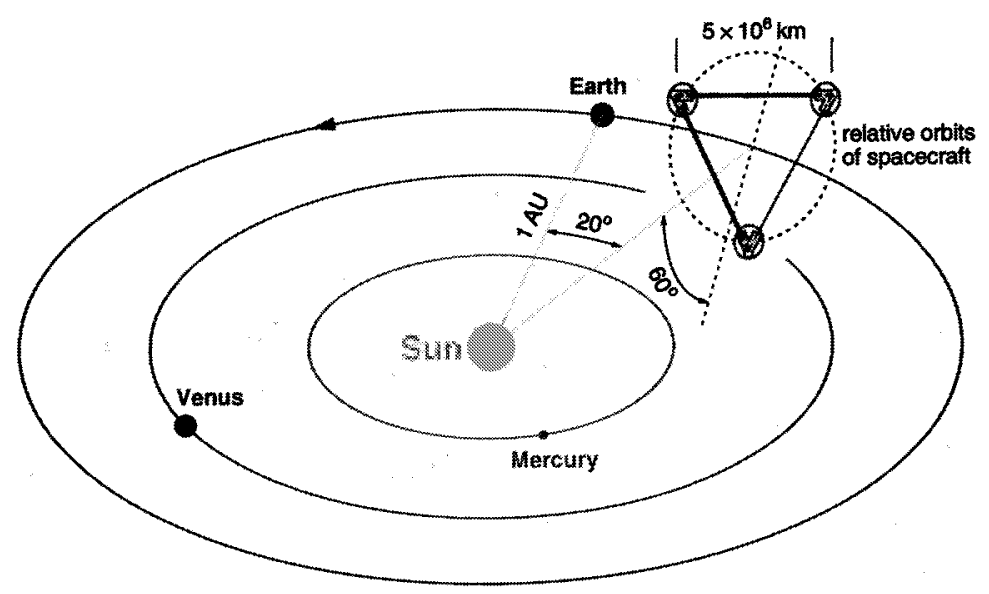

FIGURE 4. LISA configuration: three spacecraft in an equilateral triangle. Drawing not to scale: the LISA triangle is drawn one order of magnitude too large.

While LISA can be described as a big Michelson interferometer, the actual implementation in space is very different from a laser interferometer on the ground and is much more reminiscent of the technique called spacecraft tracking, but here realized with infrared laser light instead of radio waves. The laser light going out from the center spacecraft to the other corners is not directly reflected back because 
very little light intensity would be left over that way. Instead, in complete analogy with an RF transponder scheme, the laser on the distant spacecraft is phase-locked to the incoming light providing a return beam with full intensity again. After being transponded back from the far spacecraft to the center spacecraft, the light is superposed with the on-board laser light serving as a local oscillator in a heterodyne detection. This gives information on the length of one arm modulo the laser frequency. The other arm is treated the same way, giving information on the length of the other arm modulo the same laser frequency. The difference between these two signals will thus give the difference between the two arm lengths (i.e. the gravitational wave signal). The sum will give information on laser frequency fluctuations.

Each spacecraft contains two optical assemblies. The two assemblies on one spacecraft are each pointing towards an identical assembly on each of the other two spacecraft to form a Michelson interferometer. A $1 \mathrm{~W}$ infrared laser beam is transmitted to the corresponding remote spacecraft via a $30-\mathrm{cm}$ aperture $f / 1$ Cassegrain telescope. The same telescope is used to focus the very weak beam (a few $\mathrm{pW}$ ) coming from the distant spacecraft and to direct the light to a sensitive photodetector where it is superimposed with a fraction of the original local light. At the heart of each assembly is a vacuum enclosure containing a free-flying polished platinum-gold cube, $4 \mathrm{~cm}$ in size, referred to as the proof mass, which serves as an optical reference ("mirror") for the light beams. A passing gravitational wave will change the length of the optical path between the proof masses of one arm of the interferometer relative to the other arm. The distance fluctuations are measured to sub-Ångstrom precision which, when combined with the large separation between the spacecraft, allows LISA to detect gravitational-wave strains down to a level of order $\Delta \ell / \ell=10^{-23}$ in one year of observation, with a signal-to-noise ratio of 5 .

The spacecraft mainly serve to shield the proof masses from the adverse effects due to the solar radiation pressure, and the spacecraft position does not directly enter into the measurement. It is nevertheless necessary to keep all spacecraft moderately accurately $\left(10^{-8} \mathrm{~m} / \sqrt{\mathrm{Hz}}\right.$ in the measurement band) centered on their respective proof masses to reduce spurious local noise forces. This is achieved by a "drag-free" control system, consisting of an accelerometer (or inertial sensor) and a system of electrical thrusters.

Capacitive sensing in three dimensions is used to measure the displacements of the proof masses relative to the spacecraft. These position signals are used in a feedback loop to command micro-Newton ion-emitting proportional thrusters to enable the spacecraft to follow its proof masses precisely. The thrusters are also used to control the attitude of the spacecraft relative to the incoming optical wavefronts, using signals derived from quadrant photodiodes. As the three-spacecraft constellation orbits the Sun in the course of one year, the observed gravitational waves are Doppler-shifted by the orbital motion. For periodic waves with sufficient signal-to-noise ratio, this allows the direction of the source to be determined to arc minute or degree precision, depending on source strength.

Each of the three LISA spacecraft has a launch mass of about $400 \mathrm{~kg}$ (plus mar- 


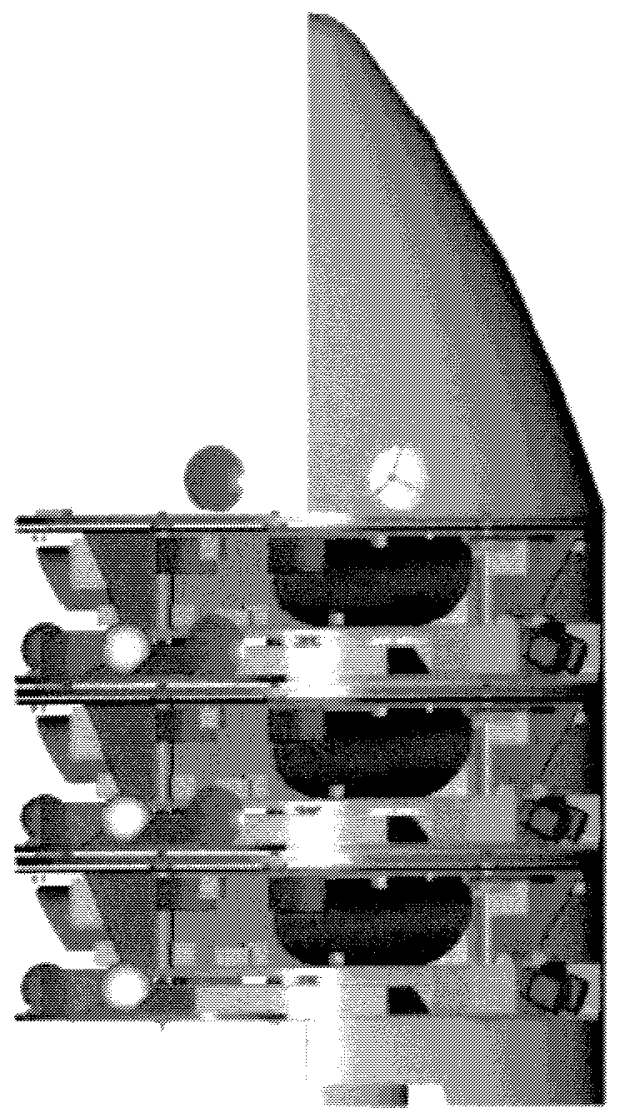

FIGURE 5. 3 LISA composite spacecraft in the Delta II $10 \mathrm{ft}$ fairing.

gin) including the payload, ion drive, all propellants and the spacecraft adapter. The ion drives are used for the transfer from the Earth orbit to the final position in interplanetary orbit. All three spacecraft can be launched by a single Delta II $7925 \mathrm{H}$. Each spacecraft carries a $30 \mathrm{~cm}$ steerable antenna used for transmitting the science and engineering data, stored on board for two days, at a rate of $7 \mathrm{kBps}$ in the X-band to the 34-m network of the DSN. Nominal mission lifetime is two years, but consumables are sized for an extended mission of more than 10 years.

LISA is envisaged as a NASA/ESA collaborative project, with NASA providing the launch vehicle, mission and science operations and about $50 \%$ of the payload, ESA providing the three spacecraft including the ion drives, and European insti- 
tutes, funded nationally, providing the other $50 \%$ of the payload. The collaborative NASA/ESA LISA mission is aimed at a launch in the 2010 time frame. LISA is a Cornerstone mission in ESA's Future Science Program Horizons 2000 and has recently been included in NASA's Roadmap.

\section{REFERENCES}

1. A.G. Lyne and D.R. Lorimer, Nature 369 (1994) 127.

2. A.V. Tutukov and L.R. Yungelson, MNRAS 260 (1993) 675.

3. C.W. Misner, K.S. Thorne, and J.A. Wheeler, Gravitation (Freeman \& Co., San Francisco, 1973).

4. P.R. Saulson, Fundamentals of Interferometric Gravitational Wave Detectors (World Scientific, Singapore, 1994).

5. B.F. Schutz, A First Course in General Relativity (Cambridge University Press, Cambridge, 1985).

6. K.S. Thorne, Gravitational Radiation, in: S.W. Hawking and W. Israel, eds., 300 Years of Gravitation (Cambridge University Press, Cambridge, 1987) 330-458.

7. J. Weber, Phys. Rev. 117 (1960) 306.

8. M. Bassan, Class. Quant. Grav. Supplement A 39 (1994) 11.

9. F.A.E. Pirani, Acta Physica Polonica 15 (1956) 389.

10. M.E. Gertsenshtein and V.I. Pustovoit, JETP 16 (1963) 433.

11. R. Weiss, Quarterly Progress Report of RLE, MIT 105 (1971) 54.

12. G.E. Moss, L.R. Miller, and R.L. Forward, Appl. Opt. 10 (1971) 2495.

13. A. Abramovici et al., Science 256 (1992) 325.

14. G. Bradaschia et al., Nucl. Instrum. and Methods A 289 (1990) 518.

15. K. Danzmann et al., GEO600-A $300 \mathrm{~m}$ laser-interferometric gravitational wave antenna, Proc. $1^{\text {st }}$ Edoardo Amaldi Conference, Frascati, June 1994; and also: J. Hough et al., Proc. MG7, Stanford, July 1994.

16. K. Tsubono and TAMA collaboration, TAMA Project in: K. Tsubono, M.-K. Fujimoto, K. Kuroda, (Eds.), Gravitational Wave Detection, Proc. TAMA Intern. Workshop, Nov. 1996, p. 183-191, Universal Academy Press (Tokyo, 1997).

17. V.M. Kaspi, J.H. Taylor, M.F. Ryba, Astrophys. J. 428 (1994) 713. 